\title{
Integration of Male Mating and Feeding Behaviors in Caenorhabditis elegans
}

\author{
Todd R. Gruninger, Daisy G. Gualberto, Brigitte LeBoeuf, and L. Rene Garcia \\ Department of Biology, Texas A\&M University, College Station, Texas 77843-3258
}

The Caenorhabditis elegans male must integrate various environmental cues to ensure proper execution of mating. One step of male mating, the insertion of the male copulatory spicules into its mate, requires UNC-103 ERG (ether-a-go-go-related gene)-like $\mathrm{K}^{+}$channels. unc-103(lf) alleles cause males to protract their spicules spontaneously in the absence of mating cues. To identify proteins that work with UNC-103, we suppressed $u n c-103$ (lf) and isolated lev-11(rg1). LEV-11 (tropomyosin) regulates the spicules directly by controlling the male sex muscles and indirectly by controlling the pharyngeal muscles. lev-11-mediated suppression requires the pharyngeal NSM neurosecretory motor neurons; ablating these neurons in lev-11(rg1); unc-103(lf) males restores spontaneous spicule protraction. Additionally, unc-103-induced spicule protraction can be suppressed by reducing a pharyngeal-specific troponin T. These observations demonstrate that non-genitalia cells involved in feeding also mediate male sexual behaviors.

Key words: lev-11; tropomyosin; C. elegans; mating behavior; unc-103; ERG K ${ }^{+}$channel

\section{Introduction}

Nervous systems of metazoans are designed to execute specific behaviors necessary for survival and reproduction at the appropriate times. A behavior must be monitored at each step to ensure proper initiation, execution, and completion. Caenorhabditis elegans male mating behavior is ideal for addressing how a complex behavior is regulated within a simple nervous system. This complex behavior can be broken down into the cellular circuits and molecules involved in its regulation, thereby revealing basic principles in nervous-system design and function.

Male mating behavior initiates when sensilla in the male tail contact a hermaphrodite. The male presses the ventral portion of his tail against his mate and moves backward, scanning for her vulva. If he reaches the posterior or anterior ends of the hermaphrodite without encountering the vulva, he performs a ventral turn and scans the other side. When he locates the vulva, he stops backward locomotion and attempts to breach her vulva with his two copulatory spicules located in his tail. Each spicule is attached to two retractor muscles that hold the spicules inside the tail and two protractor muscles that thrust the spicules out during mating. These muscles rhythmically contract at $7-9 \mathrm{~Hz}$ causing the tips of the spicules to prod the vulval slit rapidly. When the spicules successfully breach the vulva, the protractors maintain a sustained contraction, which keeps the spicules inserted until sperm transfer is completed (Ward and Carrel, 1979; Sulston et al., 1980; Loer and Kenyon, 1993; Barker, 1994; Liu and Sternberg, 1995; Garcia et al., 2001).

Received Aug. 10, 2005; revised Nov. 7, 2005; accepted Nov. 7, 2005

This work was supported by Public Health Service Grant GM070431-1 and by the Searle Scholars Program. We thank the Caenorhabditis Genetics Center for providing strains.

Correspondence should be addressed to Dr. L. Rene Garcia, Department of Biology, Texas A\&M University, 3258 TAMU, College Station, TX 77843-3258. E-mail: rgarcia@mail.bio.tamu.edu.

DOI:10.1523/JNEUROSCI.3364-05.2006

Copyright $\odot 2006$ Society for Neuroscience $\quad$ 0270-6474/06/260169-11\$15.00/0
Previously, we isolated an allele of unc-103 that causes males to display the spicule insertion step of mating behavior in the absence of hermaphrodite mating cues (Garcia and Sternberg, 2003). unc-103 encodes an ether-a-go-go-related gene (ERG)like voltage-gated potassium channel that is expressed broadly in males and hermaphrodites; however, the only gross phenotype that unc-103(sy557) males display is premature spicule protraction before and during mating behavior. Similarly, the hermaphrodite counterparts have a subtle precocious egg-laying defect, suggesting that this gene regulates the appropriate timing of sexrelated behaviors. The spontaneous spicule protraction associated with unc-103(sy557) requires both SPC motor neurons that innervate the protractor muscles and the auxiliary extension of the dorsal protractors, the anal depressor muscle. The loss-offunction allele unc-103(sy557) contains two point mutations that cause $80 \%$ of males to protract their spicules prematurely, whereas only $40 \%$ of males carrying a deletion allele of unc-103 display the phenotype. We have suggested previously that the sy557 allele might artificially interfere with other pathways that control spicule protraction (Garcia and Sternberg, 2003).

In this study, we use unc-103(lf) mutations to investigate how cells involved in a specialized behavior can be influenced by cells used in a general behavior. We find a distinct regulatory connection between the pharynx, the major function of which is feeding, and the mating-specific spicule muscles. We propose that before mating, reduced pharyngeal muscle activity signals the neurosecretory motor (NSM) neurons to downregulate excitable cells in the male genitalia.

\section{Materials and Methods}

Strains. All strains used in this study contained him-5(e1490) on LGV (Hodgkin et al., 1979) and were maintained using standard culture conditions (Brenner, 1974). The wild-type reference strain used was N2 Bristol (Brenner, 1974). We also used the following alleles in this study: egl-30(tg26gf) (Moghal et al., 2003), unc-75(e95) (Brenner, 1974), sy558 
(Garcia and Sternberg, 2003), and lev-11(x12) (Lewis et al., 1980) on LGI; unc-103(sy557) (Garcia and Sternberg, 2003), unc-103(n1213) (Park and Horvitz, 1986), unc-103(e1597) (Hodgkin, 1983), pha-1(e2123) (Schnabel and Schnabel, 1990), and unc-64(e246) (Brenner, 1974) on LGIII; egl-19(n2386gf) (Lee et al., 1997), and sy574 (Garcia and Sternberg, 2003) on LGIV; unc-60(m35) (Ono et al., 1999) on LGV. The Hawaiian CB4856 strain was used as the source of polymorphisms for single nucleotide polymorphism (SNP) mapping (Wicks et al., 2001).

Mapping of lev-11(rg1) and lev-11(sy558). Details of lev-11(sy558) isolation have been described previously (Garcia and Sternberg, 2003). To generate lev-11(rg1), we first ethylmethane sulfonate-mutagenized unc103(sy557); him-5 hermaphrodites (Brenner, 1974); from 7500 mutagenized gametes, we picked $50 \mathrm{~F} 2$ hermaphrodites that displayed abnormal behaviors such as increased or decreased locomotion, defective egg laying, reduced feeding, and decreased defecation behavior. From these hermaphrodites, we scored the male progeny to determine whether they were also suppressed for unc-103-mediated spicule protraction; only three lines were suppressed for the abnormal spicule behavior. We then tested whether males from the three lines could mate with unc-64(e246); only rg1; unc-103(sy557) males could sire progeny.

rg1 and sy558 were both mapped to LGI, near the gene lev-11, using standard genetic means. Sequence analysis of $l e v-11$ exons found $r g 1$ and sy 558 to be changes in exon 7 and exon $4 \mathrm{~b}$, respectively. The rg1 lesion changes the sequence CGTACGAGG to CGTACAAGG, and the sy558 lesion changes the sequence GGATGAGGAGCG to GGATGAGAAGCG.

Assay for protraction constitutive males. To observe the number of males that spontaneously protract their spicules, we separated L4 males from hermaphrodites to a plate seeded with Escherichia coli OP50. Twenty-four hours later, we scored the males for the protraction constitutive (Prc) phenotype with a Wild M5A microscope. To measure the effects of liquid media on various Prc mutants, we placed 10 L4 males in microfuge tubes containing S-medium with OP50. To assay effects of starvation on the Prc phenotype, we washed L4 males with M9 buffer and placed them on a fresh nematode growth medium (NGM) plate without food to ensure all traces of bacteria were removed completely. These males were then placed in microfuge tubes containing $20 \mu \mathrm{l}$ of $\mathrm{S}$-medium. Control males were placed in a $20 \mu \mathrm{l} \mathrm{S}$-medium solution containing OP50. For all liquid culture assays, we removed adult males to fresh NGM plates after $24 \mathrm{~h}$ and scored them for the Prc phenotype.

Mating observation. We observed mating with a Stemi SV11 dissecting microscope (Zeiss, Thornwood, NY). We placed 10 unc-64(e246) hermaphrodites and one male (all $15-24 \mathrm{~h}$ old) on a $10 \mathrm{~mm}$ plate seeded with a $5 \mathrm{~mm}$ OP50 lawn. To assay each male's drive to mate, we recorded the number of hermaphrodites a male encountered before he initiated mating behavior. Males who required three or more encounters were considered defective. A male was considered defective in turning if he failed to complete a successful turn within three attempts after reaching the end of the hermaphrodite. A male was considered vulva location defective if he required three or more contacts with a hermaphrodite vulva before attempting to insert his spicules.

Pharmacology. We dissolved levamisole (ICN Biomedicals, Aurora, $\mathrm{OH})$ in distilled water to make a $100 \mathrm{~mm}$ stock solution. The stock was then serially diluted in water as needed. One milliliter of the drug was added to a 3-well Pyrex titer dish, and 10 virgin adult males ( $<24 \mathrm{~h}$ old) were placed in the drug. We observed the males for $2 \mathrm{~min}$ with a Wild M5A microscope. The males were considered resistant to the drug if they
Primer sequence

\begin{tabular}{|c|c|}
\hline Target & Primer sequence \\
\hline \multirow[t]{2}{*}{ tnt-1, exon 5} & GCTAAGGGAGGAAAGGATGTCATGAGCAAGG \\
\hline & GTCATAGTCTTGACGTTCACGTCTCTTCTCC \\
\hline \multirow[t]{2}{*}{ tnt-2, exon 5} & AAAGAACCTTGAACCACCAAAGTACGT \\
\hline & AGCTCCCTCATTTTGGCTTTGAACTGG \\
\hline \multirow[t]{2}{*}{ tnt-3, exon 5} & GCAGCCAAACGCCGTCACGAGGAAG \\
\hline & TTCTTCTTGACGACGACGGTCATCAGCAGCACGA \\
\hline \multirow[t]{2}{*}{ tnt-4, exon 3} & CGGAGAAGCTCTAAATGAAGGTGAG \\
\hline & CGCTCGGCAGCTTCACGCTCTTCTTGC \\
\hline T7 promoter sequence ${ }^{a}$ & TTAATACGACTCACTATAGGGAGA \\
\hline \multirow[t]{2}{*}{ unc-103 promoter } & GGATCAGAATCACGTCATGCTGCAATCCAATTAGC \\
\hline & TGCCACGTGGTTGTGTG-TGTGTACCC \\
\hline \multirow[t]{2}{*}{ unc-103 promoter and coding } & GCGCGCTAGCGGATCAGAATCACGTCATGCTGCAATCCAATTAGC \\
\hline & GCGCGCTAGCTAGAATAGTATCAGTTTCTTGTGTGG \\
\hline \multirow[t]{2}{*}{ tnt- 4 promoter and coding } & GTTCTCATAAACCTGACGGCGCTCCTT \\
\hline & CCAAAATTAGCTCAATTGATCAAATAACTG \\
\hline \multirow[t]{2}{*}{ tph-1 promoter } & GGTTTTCACACAGCTTTGGGTATTC \\
\hline & TGCGGATGCCATCTGAAACAACG \\
\hline \multirow[t]{2}{*}{ tph-1 promoter, attB flanked } & GGGGACAAGTTTGTACAAAAAAGCAGGCTTCGGTTTTCACACAGCTTTGGGTATTC \\
\hline & GGGACCACTTTGTACAAGAAAGCTGGGTCTGCGGATGCCATCTGAAACAACG \\
\hline \multirow[t]{2}{*}{ lev-11 body-wall/sex-muscle promoter } & CACAGCGATGATGTGTCATGGCTTCC \\
\hline & GCCTGCATCTTCTTCTTAATGGCGTCCAT \\
\hline \multirow[t]{2}{*}{ lev-11 pharyngeal/intestine promoter } & ACTAGTCACTTGGCTTCACCCAGATGGG \\
\hline & CAACCATGGCGAGCTTACGGGC \\
\hline \multirow[t]{2}{*}{ lev-11 promoter and coding (12 kb) } & CACAGCGATGATGTGTCATGGCTTCC \\
\hline & CAGGACTGTTGATATTGCATGGCCCTGAA \\
\hline lev-11 promoter to exon 3a (primer 1) & CACAGCGATGATGTGTCATGGCTTCC \\
\hline lev-11 promoter to exon 3a (primer 2) & GGAGAAGGAGAAGACCGTTCAAGAGGCTGAGGCTGAGGTCGCTTCTTTGA \\
\hline lev-11 exon 4b to 3'UTR (primer 3) & CAGGACTGTTGATATTGCATGGCCCTGAA \\
\hline lev-11 exon 4b to 3' UTR (primer 4) & TCAAAGAAGCGACCTCAGCCTCAGCCTCTTGAACGGTCTTCTCCTTCTCC \\
\hline \multirow[t]{2}{*}{ lev-11 pharyngeal promoter to $3^{\prime}$ UTR } & ACTAGTCACTTGGCTTCACCCAGATGGG \\
\hline & CAGGACTGTTGATATTGCATGGCCCTGAA \\
\hline
\end{tabular}

CAGGACTGTTGATATTGCATGGCCCTGAA

Table 1. Primers used in this study

were not paralyzed after $2 \mathrm{~min}$. To measure the sensitivity of the spicule muscles to levamisole, five virgin adult males were added to the drug and observed for $5 \mathrm{~min}$. We scored the males as sensitive to the drug if they kept their spicules protracted for at least $10 \mathrm{~s}$. We estimated the $\mathrm{EC}_{50}$ and $\mathrm{EC}_{90}$ values by first converting the percentages of protracted males to probit values (Finney, 1971). We then plotted the probit values against the log of levamisole concentration, fitted the curve using linear regression (least-squared tests method), and estimated the $\mathrm{EC}_{50}$ value from the fitted probit of 5 and the $\mathrm{EC}_{90}$ value from the fitted probit of 6.28 .

RNA-mediated interference soaking. To generate double-stranded RNA (dsRNA), we PCR amplified individual exons of tnt-1, tnt-2, tnt-3, and tnt-4 from N2 genomic DNA. We then flanked each exon with T7 promoter sequences using T7 primers. Primer sequences are listed in Table 1. PCR products were then used for in vitro transcription with a T7MEGAshortscript kit from Ambion (Austin, TX). Recovery of purified dsRNA was obtained following the protocol supplied with the kit. The final RNA concentration for each reaction was $\sim 5 \mu \mathrm{g} / \mu \mathrm{l}$.

To perform RNA-mediated interference (RNAi), we placed L4 stage males in microfuge tubes containing a solution of $5 \mu \mathrm{l}$ of dsRNA, $13 \mu \mathrm{l}$ of $\mathrm{S}$-medium, and $2 \mu \mathrm{l}$ of concentrated OP50 as the food source. Control worms were placed in a solution containing only S-medium and OP50. After $24 \mathrm{~h}$, we removed the adult males to a fresh NGM plate and immediately scored them for the Prc phenotype.

Plasmids. All primers and sequences are listed in Table 1. pDG9 contains green fluorescent protein (GFP) expressed from sequences $3.5 \mathrm{~kb}$ upstream of the first lev-11 exon. We PCR amplified these lev-11 sequences and blunt-end ligated this fragment into the SmaI site of pPD95.77 (plasmid courtesy of A. Fire, Stanford University School of Medicine, Stanford, CA). pTG13 contains cyan fluorescent protein (CFP) expressed from lev-11 sequences including $1.3 \mathrm{~kb}$ upstream of exon $3 b$ to exon 6. We PCR amplified these sequences using primers flanked with attB sites and recombined the product into pDONR221, a donor vector from Invitrogen (San Diego, CA), to produce pTG12. 
pTG13 was then generated by recombining pTG12 into pGW77C. pGW77C is a plasmid derived from pSX77CFP (plasmid courtesy of $\mathrm{N}$. Moghal, Huntsman Cancer Institute, University of Utah, Salt Lake City, UT) containing the Gateway cloning cassette. pDG9 was injected into the germ line of him-5 hermaphrodites at a concentration of $5 \mathrm{ng} / \mu \mathrm{l}$, and pTG13 was injected at $2 \mathrm{ng} / \mu \mathrm{l}$ (Mello et al., 1991). Three lines from each injection were analyzed for lev-11 expression. To generate pLR10, we PCR amplified an $8.7 \mathrm{~kb}$ region between the first unc-103 exon and the preceding gene C30D11.2. We then blunt-end ligated this PCR fragment into the SmaI site of the plasmid pSX77YFP, a vector derived from pPD95.77 that contains yellow fluorescent protein (YFP) in place of GFP (plasmid courtesy of N. Moghal). We injected a mixture containing pLR10 (10 ng/ $\mu \mathrm{l}), \mathrm{pBX} 1(100 \mathrm{ng} / \mu \mathrm{l})$, and pUC18 $(90 \mathrm{ng} / \mu \mathrm{l})$ into the germ line of pha-1; him-5 hermaphrodites (Granato et al., 1994). Three transmitting lines were then analyzed for unc-103 expression. Cells were identified using drawings from the studies of Sulston et al. (1980) and White et al. (1986). To observe unc-103 sex-muscle expression, we first PCR amplified $\sim 16 \mathrm{~kb}$ of DNA containing the $8.7 \mathrm{~kb}$ region upstream of the first unc-103 exon and all coding sequences excluding the stop codon. We cut the ends of this PCR fragment with NheI and ligated it to XbaIdigested pSX77YFP, so that the unc-103 coding sequence was in frame with YFP. We found this construct to be unstable in E. coli; therefore, we injected the ligation mixture directly into $C$. elegans. To generate transgenic worms, we coinjected the $16 \mathrm{~kb}$ unc-103-XbaI-digested pSX77YFP mixture $(200 \mathrm{ng} / \mu \mathrm{l})$ with pBX1 (100 ng/ $\mu \mathrm{l})$ into $u n c-103(n 1213)$ pha-1; him-5 hermaphrodites. We obtained seven transmitting lines. All lines were fluorescent, suppressed for the Prc phenotype, lethargic, and had reduced egg-laying behavior, suggesting that the transgene was overexpressed. We analyzed worms carrying the transgene array rgEx20 (see Fig. 1). When the ligation mixture was injected at $100 \mathrm{ng} / \mu \mathrm{l}$, the synthetic phenotypes were reduced, but the fluorescence was also too dim to identify cells.

To generate pTG7, we PCR amplified a $6.2 \mathrm{~kb}$ fragment of DNA that covers $3 \mathrm{~kb}$ upstream of the start codon and exons $1-5$ of $t$ tht 4 . pPD95.79 (plasmid courtesy of A. Fire) was cut with ApaI, FspI, and HindIII, and the $2000 \mathrm{bp}$ fragment was retained. pBR322 was cut with AvaI and Hin$d \mathrm{III}$; the $2953 \mathrm{bp}$ fragment was retained and blunt-end ligated to the pPD95.79 fragment above to make pTG5. We then blunt-end ligated the tnt-4 PCR fragment into the SmaI site of pTG5 to make the plasmid pTG7. GFP is fused in frame to the last exon of the smaller of the two reported tnt-4 isoforms (www.wormbase.org). pTG7 (20 ng/ $\mu \mathrm{l})$, pBX1 $(100 \mathrm{ng} / \mu \mathrm{l})$, and pUC18 $(80 \mathrm{ng} / \mu \mathrm{l})$ were injected into the germ line of pha-1; him-5 hermaphodites (Mello et al., 1991; Granato et al., 1994). We analyzed 20 mid-L4 and 20 adult males from two transgenic lines for tnt-4 expression.

To generate pTG11, we amplified sequences $1 \mathrm{~kb}$ upstream of the $t p h-1$ start codon (Sze et al., 2000). This sequence was re-amplified using the same primers flanked with attB sites. This was then recombined into pDONR221 to generate pTG9. pTG9 was then subsequently recombined into pGW77C to make pTG11.pTG11 (50 ng/ $\mu \mathrm{l})$, pBX1 (100 ng/ $\mu \mathrm{l})$, and pDONR221(50 ng/ $\mu \mathrm{l}$; Invitrogen) were injected into the germ line of pha-1; him-5 hermaphrodites.

Injections of lev-11 genomic sequences. We PCR amplified a $12 \mathrm{~kb}$ DNA fragment from $\mathrm{N} 2$ or sy558 DNA that contains $3.5 \mathrm{~kb}$ of $l e v-11$ upstream sequences, all of the $l e v-11$ exons, and the $3^{\prime}$ untranslated region (UTR) (Kagawa et al., 1995). To rescue lev-11(rg1) in all muscles, we injected a mixture containing lev-11(+) PCR product $(2 \mathrm{ng} / \mu \mathrm{l}), \mathrm{pBX} 1(100 \mathrm{ng} / \mu \mathrm{l})$, and pUC18 (98 ng/ $\mu \mathrm{l}$ ) into lev-11(rg1); unc-103(sy557) pha-1; him-5. We obtained 16 transmitting lines, and only two were rescued for gross lev11(rg1) phenotypes such as defective egg-laying behavior, subtle bodywall muscle spasms, and levamisole resistance; one of these lines containing the array $\operatorname{rgEx} 9$ was scored for the Prc phenotype. Injections that contain $l e v-11(+)$ DNA at concentrations $\geq 5 \mathrm{ng} / \mu \mathrm{l}$ resulted in larval lethality or artificially hyper-contracted animals. To phenocopy lev11(sy558) worms, we injected lev-11(sy558) PCR product (100 pg/ $\mu \mathrm{l})$, pBX1(100 ng/ $\mu \mathrm{l})$, and pUC18(99 ng/ $\mu \mathrm{l})$ into pha-1; him-5. We obtained 18 transmitting lines, and only one line produced males that phenocopied sy558 animals. Injections that contained lev-11(sy558) DNA at concentrations $\geq 200 \mathrm{pg} / \mu \mathrm{l}$ resulted in embryonic and larval lethality.
The lev-11 gene has been reported to contain two groups of isoforms: those that express in the body-wall, anal, and sex muscle and those that express in the pharyngeal muscle and intestine (Kagawa et al., 1995; Anyanful et al., 2001). To omit the promoter and exons specific to the intestine and pharyngeal forms, we used four specific primers. Primers 1 and 2 were used to amplify sequences containing $3.5 \mathrm{~kb}$ upstream of the start codon down to exon 3a. Primer 2 also contained homology to the initial sequence of exon $4 \mathrm{~b}$. Primers 3 and 4 were used to amplify sequences containing exon $4 \mathrm{~b}$ down to $475 \mathrm{bp}$ of the $3^{\prime}$ UTR. Primer 4 contained homology to the exon 3a-specific sequences of primer 2 . The two PCR products were then annealed, extended, and subsequently amplified using primers 1 and 3. The body-wall, anal, and sex musclespecific construct $(2 \mathrm{ng} / \mu \mathrm{l}), \mathrm{pBX} 1(100 \mathrm{ng} / \mu \mathrm{l})$, and the GFP marker plasmid pDG9 $(5 \mathrm{ng} / \mu \mathrm{l})$ were injected into the germ line of lev-11(rg1); unc-103(sy557) pha-1; him-5 hermaphrodites. To rescue lev-11(rg1) in the pharyngeal muscle and intestine, we PCR amplified a $6 \mathrm{~kb}$ DNA fragment from N2 DNA that contains $1.3 \mathrm{~kb}$ of sequences upstream of lev-11 exon 3b, exons 3b to 9c, and the 3' UTR (Kagawa et al., 1995). We injected the pharyngeal muscle- and intestine-specific construct at 200 $\mathrm{pg} / \mu \mathrm{l}$ with pBX1 $(100 \mathrm{ng} / \mu \mathrm{l})$ and the myo-2::GFP plasmid pPD118.33 (plasmid courtesy of A. Fire). Injections that contained the pharyngeal/ intestine construct at concentrations $\geq 200 \mathrm{pg} / \mu \mathrm{l}$ resulted in larval arrest. We obtained seven lines for both sets of injections; rgEx26 [body-wall/ anal/sex muscle lev-11(+)] and rgEx27 [pharyngeal/intestine lev-11(+)] were further analyzed.

To obtain lev-11(rg1); unc-103(n1213); him-5 animals with rgEx26 or rgEx27, we crossed lev-11(rg1); unc-103(sy557) pha-1; him-5; rgEx26 and lev-11(rg1); unc-103(sy557) pha-1; him-5; rgEx27 males into pha-1; unc103(e1597gf); him-5 hermaphrodites. F2 animals homozygous for lev11 (rg1) and unc-103(e1597) were kept, and hermaphrodites still expressing the GFP marker were then crossed with lev-11(rg1); unc-103(n1213); him-5 males. We repulsed unc-103(e1597gf) to obtain lev-11(rg1); unc103(n1213); him5; rgEx26 and lev-11(rg1); unc-103(sy557); him-5; rgEx27; fluorescing transgenic males were then assayed for the Prc phenotype.

Laser ablations. We used standard laser ablation protocols to kill the pair of NSM neurons in the male pharynx (Bargmann and Avery, 1995). We operated on L3 stage males and also placed nonoperated L3 males on a pad soaked with anesthetic to rule out any effects it may have on behavior. The locations of the NSM neurons were verified by the pTG11 tph-1::CFP reporter construct.

To assay the effects of cell ablation on the Prc phenotype and pharyngeal pumping rate, we allowed animals from each experiment to recover for $2 \mathrm{~d}$ on NGM plates seeded with OP50 and scored them for the phenotype. We measured effects on pharyngeal pumping by counting the number of posterior bulb contractions in 1 min using a Stemi SV11 microscope (Zeiss).

\section{Results \\ unc-103 is expressed in the sex muscles and broadly in the nervous system}

To understand how C. elegans males regulate their spicule muscles, we previously isolated mutations that cause males to protract their spicules spontaneously in the absence of hermaphrodites (Garcia and Sternberg, 2003). From this screen, we isolated the dominant loss-of-function allele unc-103(sy557). The only obvious abnormal phenotype that sy557 and the deletion mutation $u n c-103(n 1213)$ cause is premature spicule protraction before and during mating behavior. In contrast, the neomorphic unc-103(e1597gf) allele causes broad abnormal behaviors in both sexes such as a reduction in locomotion, egg laying, male mating, defecation, and pharyngeal pumping, indicating that UNC-103 channels are present in cells that control many worm behaviors (Park and Horvitz, 1986; Reiner et al., 1999; Garcia and Sternberg, 2003; Petersen et al., 2004).

An $u n c-103::$ GFP translational fusion containing sequences 5 $\mathrm{kb}$ upstream of the $u n c-103$ start codon expresses in the SPC and 
PCB cholinergic motor neurons that regulate spicule muscle contraction. This translational fusion also expressed in the AS ventral cord neurons as well as the ALA, ADL, ASK, AVH, AVJ, AIN, AVA, ASJ, SMDD, SIA, ADE and AVD neurons in the head (Garcia and Sternberg, 2003). However, because the fusion protein was mainly localized on the membranes of neuronal processes, we found it difficult to identify many other unc-103-expressing head neurons. To study the expression pattern in more detail, we made a construct (pLR10) that contains YFP expressed from $8.7 \mathrm{~kb}$ of DNA upstream of the unc-103 start codon. From this construct, we found that unc-103 also expresses in the pharyngeal neurons (I2 and NSM) (Fig. $1 B$ ), head neurons [IL1, IL2, OLL, URAD, ASH, AVD, AUA, and SIAV (Fig. $1 B$ ) and OLQ, RIV, URYV, AIN, and AIA (data not shown)], PCA in the postcloacal sensilla, and one of two ray neurons in rays $1,2,3,4,6$, and 9 (data not shown). We also injected an unc-103::YFP translational fusion expressed from the 8.7 $\mathrm{kb}$ upstream region and observed that the anal depressor, spicule protractor, retractor, and other male sex muscles also expressed UNC-103 (Fig. 1D); in the hermaphrodite, the vulva muscles also express the transgene (data not shown). These results indicate that unc-103 expression is broader than what we reported previously and that unc-103 is expressed in both muscles and neurons that regulate spicule protraction.

\section{Spicule protraction mutants vary in their penetrance and response to external conditions}

During the course of working with unc-103(n1213), unc103(sy557), and other Prc mutants, we noticed that the percentages of males displaying the Prc phenotype vary with temperature, availability of food, amount of moisture, and, in some cases, the density of animals on the plates. The variability of the unc103(lf)-mediated Prc phenotype suggested to us that the neurons and muscles directly controlling spicule protraction may be indirectly regulated by other cells in the worm that are sensitive to nutritional or environmental perturbations. To determine more quantitatively whether external parameters could affect the unc103(lf) phenotype, we compared the effects of growth in liquid and the abundance of food on unc-103(lf) males and as a control, also on various other Prc mutants. Two other Prc mutants, sy574 and sy558, were isolated in the original screen with unc103(sy557) (Garcia and Sternberg, 2003). Similar to unc103(sy557) and unc-103(n1213), sy574 and sy558 cause $\sim 60-$ $80 \%$ of males to display a Prc phenotype (Table 1) without obviously disrupting additional behaviors. The gain-of-function alleles egl-19(n2368gf) and egl-30(tg26gf), which affect the L-type voltage-gated calcium channel $\alpha 1$ subunit and G $\alpha \mathrm{q}$, respectively, also cause males to display the Prc phenotype (Table 2), but unlike sy574, sy558, and the unc-103 alleles, both egl-19(n2368gf) and egl-30(tg26gf) affect all behaviors (Garcia et al., 2001; Moghal et al., 2003).

To determine whether nutritional status or liquid growth al-
B

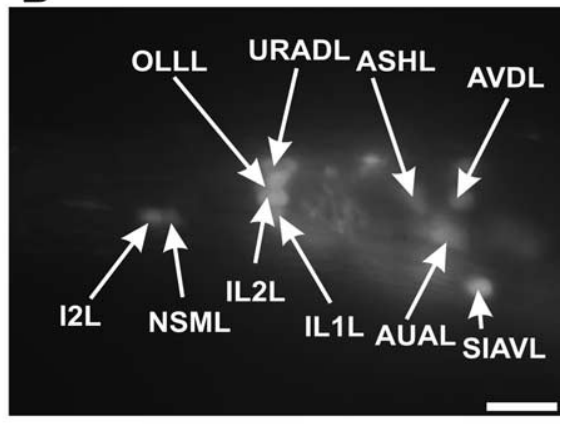

D

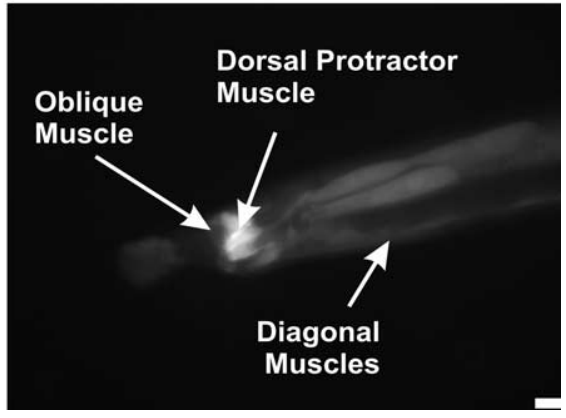

Diagonal Muscles

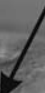

SIAVL

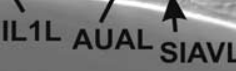

orsal Protractor

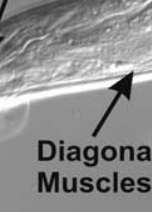

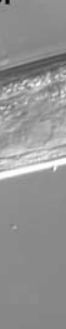

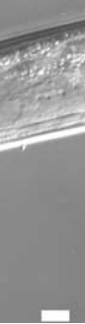

Figure 1. unc-103 expression pattern in the C. elegans male. $\boldsymbol{A}, \boldsymbol{B}$, Nomarski $(\boldsymbol{A})$ and fluorescence $(\boldsymbol{B})$ images of the left lateral 作 are labeled. $\boldsymbol{C}, \boldsymbol{D}$, Nomarski $(\boldsymbol{C})$ and fluorescence $(\boldsymbol{D})$ images of the right lateral tail region of a male expressing the unc-103::YFP translational fusion. Sex muscles in the focal plane are labeled. Scale bars, $10 \mu \mathrm{m}$.

Table 2. Prc mutants vary in their penetrance and response to external conditions

\begin{tabular}{lccc}
\hline Genotype & $\begin{array}{l}\text { Plates plus food } \\
\% \text { males } \\
\text { protracted }\end{array}$ & $\begin{array}{l}\text { S-medium plus } \\
\text { food \% males } \\
\text { protracted }\end{array}$ & $\begin{array}{l}\text { S-medium \% males } \\
\text { protracted }\end{array}$ \\
\hline Wild type & $9(66)$ & $0(20)$ & $0(20)$ \\
unc-103(sy557) & $79(61)$ & $47(32)^{*}$ & $34(32)$ \\
unc-103(n1213) & $35(20)$ & $37(30)$ & $11(20)^{* *}$ \\
sy558 & $78(144)$ & $89(18)$ & $0(10)^{* *}$ \\
egl-19(n2368gf) & $100(10)$ & $100(10)$ & $100(10)$ \\
sy574 & $58(11)$ & $73(11)$ & $73(11)$ \\
egl-30(tg26gf) & $53(12)$ & $67(12)$ & $13(16)^{* *}$ \\
\hline
\end{tabular}

The numbers in parentheses refer to the number of animals assayed.

${ }^{*} p<0.05$ compared with plates plus food; ${ }^{* *} p<0.05$ compared with S-medium plus food.

ters the protraction phenotype of each mutant, we removed L4 larval stage males from plates with food and reared them for $24 \mathrm{~h}$ in buffer that contained food (E. coli OP50) or no food. We found that the absence of food during the last larval molt can suppress the Prc phenotype in unc-103(n1213), sy558, and egl-30(tg26gf) mutants (Table 2). However, this condition has no effect on sy574 and egl-19(n2368gf), suggesting that food restriction has a specific effect on males that contain the former mutations. Unlike the other Prc mutations, we also found that rearing unc103(sy557) males in liquid culture with food partially suppresses the protraction defect, thereby possibly masking any effects mediated through the absence of food (Table 2).

Although it is not clear how these external parameters are suppressing the Prc phenotype, it appears that these mutations are disrupting regulation of the spicules in different ways. We hypothesize that there are compensating mechanisms activated in these different conditions that can inhibit spontaneous spicule protraction in certain genetic backgrounds. Consequently, we reasoned we could identify molecules activated in these different 
Table 3. lev-11 can be altered to activate or suppress the Prc phenotype

\begin{tabular}{lrr}
\hline Genotype & $\%$ Males protracted & $n$ \\
\hline Wild type & 4 & 26 \\
unc-103(sy557) & 82 & 43 \\
unc-103(n1213) & 42 & 91 \\
egl-30(tg26gf) & 53 & 36 \\
egl-19(n2368gf) & 100 & 30 \\
sy574 & 48 & 79 \\
lev-11(rg1) & 0 & 26 \\
lev-11(x12) & 0 & 20 \\
lev-11(sy558) & 78 & 144 \\
lev-11(sy558)/+ & 47 & 74 \\
lev-11(rg1); unc-103(sy557) & 17 & 126 \\
lev-11(rg1)/+; unc-103(sy557) & 42 & 41 \\
lev-11(x12); unc-103(sy557) & 13 & 72 \\
lev-11(rg1); unc-103(n1213) & 1 & 69 \\
egl-30(tg26gf) lev-11(rg1) & 13 & 45 \\
lev-11(rg1); egl-19(n2368gf) & 98 & 57 \\
lev-11(rg1); sy574 & 56 & 54 \\
lev-11(rg1)/lev-11(sy558) & 16 & 96 \\
\hline
\end{tabular}

conditions by screening for a genetic suppressor of the unc-103induced Prc phenotype.

\section{lev-11(rg1) suppresses unc-103(lf)-induced spicule protraction; lev-11(sy558) induces spicule protraction}

We performed a suppressor screen in the unc-103(sy557) background to identify what molecules when mutated can reduce unc-103(lf)-mediated spicule protraction. Animals were first screened for general abnormalities that wild type may show when deprived of food (Horvitz et al., 1982; Trent et al., 1983; Avery and Horvitz, 1990; Thomas, 1990; Sawin et al., 2000). Because we were not interested in mutations that obliterated the function of cells used in spicule insertion behavior, males from these behaviorally abnormal animals were then assayed for the ability to sire progeny, in addition to suppressing unc-103(sy557)-induced spicule protraction.

We identified the rg1 allele, which suppresses unc-103(sy557)induced protraction from $82 \%$ to $17 \%$ and $u n c-103(n 1213)$ induced protraction from $42 \%$ to $1 \%$. This allele is semidominant and can partially suppress $u n c-103$ (sy557) from $82 \%$ to $42 \%$ when heterozygous ( $\chi^{2}$ test; $p \leq 0.05$ ) (Table 3 ). We SNP mapped the $r g 1$ mutation to the right arm of linkage group 1 , near the gene $l e v-11$ (Wicks et al., 2001). lev-11 encodes the worm homolog of tropomyosin, a thin filament muscle protein that is involved in $\mathrm{Ca}^{2+}$ regulation of muscle contraction (Smillie, 1979; Taylor, 1979; Ohtsuki et al., 1986; Kagawa et al., 1995). When we separated the rg1 mutation away from unc-103(sy557), we noted that the $r g 1$ mutation confers phenotypes similar to lev-11(x12) mutants (Lewis et al., 1980; Kagawa et al., 1995). Both $r g 1$ and $x 12$ animals are longish and resistant to $1 \mathrm{~mm}$ levamisole (Lewis et al., 1980), however there are some differences between the two alleles: $r g 1$ hermaphrodites retain eggs in their uterus more than $x 12$, and the body-wall muscles of $x 12$ animals spontaneously twitch more than $\mathrm{rgl}$ animals (data not shown). Crossing lev11(x12) with rgl did not complement the $\mathrm{rg} 1$ phenotype, further suggesting that $r g 1$ is a new allele of lev-11. Additionally, lev11(x12) suppressed the Prc phenotype of unc-103(sy557) animals (Table 3).

The single tropomyosin gene in C. elegans, lev-11, gives rise to at least four tropomyosin isoforms that are expressed in different muscle types (Kagawa et al., 1995; Anyanful et al., 2001). Kagawa et al. (1995) reported previously that the four known isoforms can be classified into two groups: CeTMI and CeTMII express in the body-wall, anal, and sex muscles from a promoter region located $600-800$ bp upstream of the start codon, and CeTMIII and CeTMIV express in the pharynx and intestine from a promoter located within the third intron (Fig. $2 \mathrm{~A}$ ). We sequenced all the predicted exons of lev-11 (Kagawa et al., 1995) in the $r g 1$ background and found that $r g 1$ creates a missense mutation in exon 7. In the CeTM1 isoform, the rgl mutation changes a glutamate at amino acid 222 to a lysine in a region of tropomyosin near one of the proposed sites of regulation [troponin $\mathrm{T}$ (TNT) binding site]. Because exon 7 is found in both bodywall/sex muscle and pharyngeal muscle/intestinal isoforms of tropomyosin, this substitution should affect all muscles in the worm (Fig. 2A).

The Prc phenotype caused by the sy558 allele was also suppressible by food deprivation. Similar to the $r g 1$ allele, we SNP mapped sy558 to the right arm of linkage group 1 near lev-11. The sy558 allele is semidominant and, when heterozygous, it can cause $47 \%$ of males to display the Prc phenotype (Table 3 ). We PCR amplified the lev-11 loci from sy558 animals and injected it into wild type. Injection of $100 \mathrm{pg} / \mu \mathrm{l}$ lev-11 DNA from sy558 worms caused $55 \%$ of wild-type males $(n=49)$ to protract their spicules, whereas up to $2 \mathrm{ng} / \mu$ injected $l e v-11$ DNA from wildtype worms $(n=16)$ does not induce the Prc phenotype. We sequenced all the predicted exons of lev-11 in the sy558 background and found that $s y 558$ creates a missense mutation in exon $4 \mathrm{~b}$; in the CeTM1 isoform, this mutation results in a substitution of glutamate at amino acid 139 to lysine. Exon $4 \mathrm{~b}$ is only found in the body-wall/anal/sex muscle isoforms of tropomyosin, and therefore the lev-11(sy558) allele should not affect the pharyngeal muscles or intestine (Fig. $2 \mathrm{~A}$ ).

\section{lev-11(rg1) disrupts multiple steps of male mating behavior, except for spicule insertion}

lev-11(rg1) suppresses $u n c-103(\mathrm{lf})$ and egl-30(tg26gf) and as one copy can also suppress the semidominance of lev-11(sy558) (Table 3 ). We initially hypothesized that the altered tropomyosin may result in nonspecific weak spicule muscle functions, which was why the unc-103(sy557)- and unc-103(n1213)-mediated Prc phenotype could be suppressed. However, we have accumulated multiple observations that do not support this idea.

First, the lev-11( rg1) allele does not reduce spicule protraction caused by the egl-19(n2368gf) and sy574 alleles (Table 3), the phenotypes of which are also not affected by the absence of food (Table 2); this suggests that there is some specificity to how the altered tropomyosin acts with the different Prc mutations. lev11(rg1), as with other alleles of lev-11, confers body-wall muscle resistance to the acetylcholine agonist levamisole, hence the gene name (Lewis et al., 1980). However, we found that the $r g 1$ allele reduces the sensitivity of the spicule muscles to levamisole only slightly by a factor of 2 (Fig. 3). When we calculated the concentration of drug required to induce 50 and $90 \%$ of $l e v-11(\mathrm{rg} 1)$ and wild-type males to protract their spicules, we found that the $\mathrm{EC}_{50}$ and $\mathrm{EC}_{90}$ values for $l e v-11$ ( $r g 1$ ) were 12 and $21 \mu \mathrm{M}$, respectively, and for wild type, the $\mathrm{EC}_{50}$ and $\mathrm{EC}_{90}$ values were 5 and $9 \mu \mathrm{M}$, respectively. We concluded that the small insensitivity of the $\mathrm{rg} 1$ spicule protractor muscles to levamisole could not completely account for the suppression of unc-103-induced protraction.

Finally, one of the criteria in the isolation of the lev-11(rg1) allele was that mutant males must be able to sire progeny. To quantify the ability of lev-11(rg1) males to mate, we paired single males with paralyzed hermaphrodites. From our assay, we found that $69 \%$ of wild-type males $(n=48)$ can sire at least one prog- 
eny, whereas $31 \%$ of lev-11(rg1) males $(n=52)$ can sire at least one progeny. Because lev-11(rg1) males were less potent than wild type, we observed the mating behavior of the mutant males in greater detail to determine whether the reduction in mating potency could reveal how altered tropomyosin suppresses the unc103(lf)-induced spicule protraction. We observed male mating behavior of lev11(rg1) alone and in conjunction with the unc-103(sy557) mutation. The lev-11(rg1) allele disrupts most steps of male mating behavior, except spicule insertion behavior. We found that $78 \%$ of wild-type males will immediately initiate mating behavior once their tail comes into contact with a hermaphrodite; however, $67 \%$ of lev11( $\mathrm{rg} 1)$ males have a reduced response to tail contact with a hermaphrodite, requiring three or more contacts with hermaphrodites before initiating mating (Table 4 ). Also, $73 \%$ of $l e v-11(\mathrm{rg} 1)$ animals are inefficient at completing a turn on their first try when they reach the end of the hermaphrodite (Table 4). These males initiate the ventral turn but fail to keep their tail in contact with the hermaphrodite's cuticle. To complete an efficient turn, the male requires specialized muscle cells called the diagonal muscles; the $\mathrm{rg} 1$ lesion may be affecting these cells, reducing the ability to execute the complex movements required for turning behavior (Loer and Kenyon, 1993). During the vulva location step, lev11(rg1) males require more encounters with the vulva than wild type before attempting to insert their spicules (Table 4). Wildtype males will locate the vulva within two encounters and begin prodding the vulva with their spicules; however, lev-11(rg1) males require 3-12 encounters with the vulva before attempting to insert their spicules (Table 4). When lev-11(rg1) males eventually recognize the vulva, they prod the vulva for $132 \pm 60 \mathrm{~s}$ before their spicules breach the vulval slit ( $n=8$ males); this is not significantly different ( $t$ test; $p=0.4$ ) from wild-type males ( $n=9$ males), which require $169 \pm 107 \mathrm{~s}$ to breach the vulva. When lev-11(rg1) males $(n=5)$ do breach the vulva, they keep their spicules extended for $131 \pm 30 \mathrm{~s}$, which is the same duration as wild-type males (122 $\pm 49 \mathrm{~s} ; n=5$ males). Similarly, lev11 (x12) and lev-11(sy558) males showed no significant difference to wild type when observed for these two spicule insertion steps (data not shown). lev-11(x12) males $(n=10)$ conferred similar phenotypes to lev-11(rg1) in the other mating steps observed, with the exception that lev-11(x12) males rapidly twitched their body-wall muscles throughout mating; lev-11(sy558) males $(n=$ 10) showed no significant difference to wild type in response, turning, and vulva location behaviors (data not shown).

In contrast to lev-11(rg1), when we observed mating behavior of lev-11(rg1); unc-103(sy557) double mutants, we found that unc-103(sy557) can partially suppress the multiple mating defects caused by lev-11(rg1) (Table 4). This indicates that during mating, UNC-103 may be subtly regulating other behaviors in addition to spicule insertion. We hypothesize that although lev11(rg1) may be reducing muscle function in the spicule muscles
B

Body-wall/anal/sex muscle rescue construct

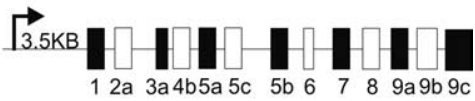

Pharyngeal muscle/intestinal rescue construct
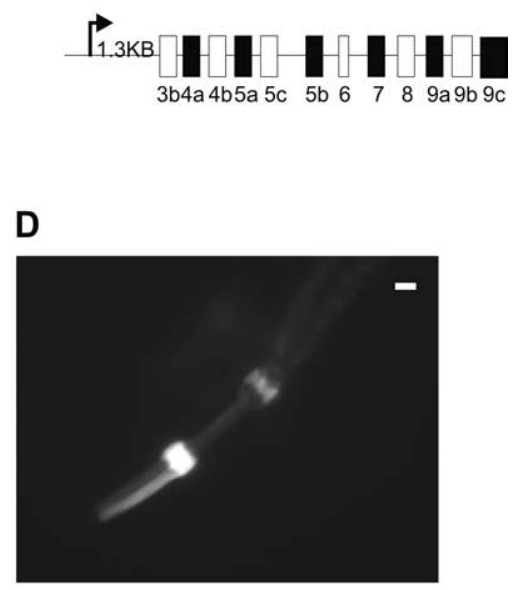

Figure 2. The lev-11 gene contains two groups of tissue-specific isoforms. $A$, The lev-11 genomic structure, highlighting the previously reported tissue-specific promoters (adapted from Anyanful et al., 2001). The locations of the sy558, rg 1, and $x 12$ alleles are shown with arrows. $\boldsymbol{B}$, The lev-11 sequences used to build tissue-specific rescue constructs. $\boldsymbol{C}$, The expression pattern of the promoter sequences that drive lev-11 in body-wall/anal/sex muscles. Scale bar, $100 \mu \mathrm{m}$. $\boldsymbol{D}$, The expression pattern of promoter sequences that drive lev-11 in the pharyngeal muscle and intestine. Scale bar, $10 \mu \mathrm{m}$.

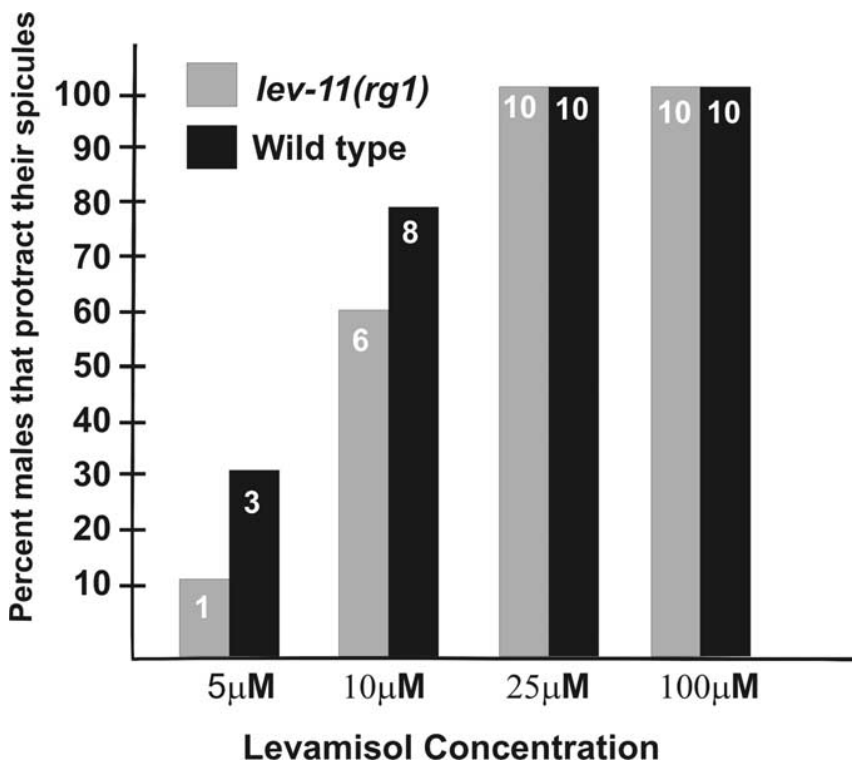

Figure 3. lev-11(rg1) mildly affects the sensitivity of the spicule muscles to levamisole. The sensitivities of $24 \mathrm{~h}$ adult lev-11(rg1) and wild-type males to different concentrations of levamisole are shown. Ten males of each genotype were assayed for each drug concentration. The numbers in the columns refer to the number of males that protract their spicules within $5 \mathrm{~min}$ of drug exposure.

at some level, it may also be perturbing some other worm function that not only reduces mating-independent spicule protraction but also reduces response to contact, vulva location, and turning behavior during mating. 
Table 4. Both body-wall/anal/sex-muscle-specific and pharyngeal/intestinal-specific lev-11 regulate male mating

\begin{tabular}{|c|c|c|c|c|c|}
\hline Relevant genotype & $\begin{array}{l}\% \text { Males } \\
\text { protracted }\end{array}$ & $\begin{array}{l}\text { \% Levamisol } \\
\text { resistant }\end{array}$ & $\begin{array}{l}\text { \% Abnormal } \\
\text { turning }\end{array}$ & $\begin{array}{l}\text { \% Abnormal } \\
\text { vulva location }\end{array}$ & $\begin{array}{l}\text { \% Abnormal } \\
\text { response }\end{array}$ \\
\hline Wild type & $9(23)$ & $0(23)$ & $13(23)$ & $13(23)$ & $22(23)$ \\
\hline unc-103(sy557) ${ }^{a}$ & $85(33)$ & n.d. & n.d. & n.d. & n.d. \\
\hline unc-103(n1213) & $35(45)$ & n.d. & n.d. & n.d. & n.d. \\
\hline lev-11(rg1) & $0(15)$ & $100(10)$ & $73(15)$ & $60(15)$ & $67(15)$ \\
\hline lev-11(rg1); unc-103(sy557) ${ }^{a}$ & $17(126)$ & $100(14)$ & $47^{* *}(34)$ & $38^{* *}(34)$ & $18^{* *}(34)$ \\
\hline lev-11(rg1); unc-103(n1213) & $1(69)$ & $100(10)$ & $50(10)$ & $40(10)$ & $50(10)$ \\
\hline lev-11(rg1); unc-103(sy557); rgEx26(body wall/anal/sex muscle lev-11(+)) ${ }^{a}$ & $35^{*}(37)$ & $39 *(18)$ & $9 *(11)$ & $45(11)$ & $0(11)$ \\
\hline lev-11(rg1); unc-103(sy557); rgEx27(pharyngeal muscle/intestine lev-11(+)) ${ }^{a}$ & $35^{*}(46)$ & $100(11)$ & $25(12)$ & $8^{*}(12)$ & $0(12)$ \\
\hline lev-11(rg1); unc-103(n1213); rgEx26(body wall/anal/sex muscle lev-11(+)) ${ }^{a}$ & $0(17)$ & $25^{*}(16)$ & n.d. & n.d. & n.d. \\
\hline lev-11(rg1); unc-103(n1213); rgEx27(pharyngeal muscle/intestine lev-11(+))a & $22^{*}(41)$ & $100(10)$ & n.d. & n.d. & n.d. \\
\hline
\end{tabular}

The numbers in parentheses refer to the number of animals assayed.

${ }^{*} p<0.05$ compared with lev-11(rg1); unc-103(sy557) or lev-11(rg1); unc-103(n1213) $\left(\chi^{2}\right.$ test); ${ }^{* *} p<0.05$ compared with lev-11(rg1) $\left(\chi^{2}\right.$ test). n.d., Not determined.

${ }^{a}$ Strains contain pha-1(e2123) and the pha-1 rescuing plasmid, pBX1.

\section{Body-wall-specific and pharyngeal-specific LEV-11 regulates spicule muscle contraction}

Because the $r g 1$ mutation maps to an exon located in all isoforms of tropomyosin, we asked which muscle type, when affected by altered tropomyosin, can suppress unc-103(sy557)- and unc103(n1213)-induced spicule protraction. Although lev-11(rg1) is semidominant (Table 3), we can inject wild-type lev-11 genomic DNA ( $r g E x 9$ ) into lev-11(rg1); unc-103(sy557) at a concentration of $2 \mathrm{ng} / \mu \mathrm{l}$ and partially restore $u n c-103(s y 557)$-mediated protraction from $12 \%(n=25)$ to $57 \%\left(n=30 ; \chi^{2}\right.$ test; $\left.p \leq 0.05\right)$. To determine where $l e v-11(\mathrm{rg} 1)$ is acting, we injected tissue-specific lev-11(+) constructs into lev-11(rg1); unc-103(sy557) hermaphrodites and assayed transmitting lines for restoration of the Prc phenotype. For body-wall, anal, and sex muscle-specific rescue, we used extension overlap PCR to amplify sequences $3.5 \mathrm{~kb}$ upstream of exon 1 and only the specific sequences found in the splicing pattern of CeTMI and CeTMII, omitting the internal promoter and exons specific to CeTMIII and CeTMIV (Fig. 2 B). For pharyngeal muscle/intestine rescue, the $1.3 \mathrm{~kb}$ internal promoter and the rest of the genomic sequences of lev-11 were amplified (Fig. 2B). The PCR products were then directly injected into lev-11(rg1); unc-103(sy557) animals. To verify the tissue specificity of the promoter sequences in each injected PCR product, we generated plasmids that contain either a transcriptional fusion of the $3.5 \mathrm{~kb}$ sequences upstream of exon 1 to GFP (pDG9) or the $1.3 \mathrm{~kb}$ sequences upstream of exon $3 \mathrm{~b}$ to CFP (pTG13) and injected these plasmids into C. elegans (Fig. 2). The $3.5 \mathrm{~kb}$ upstream sequences drove GFP expression only in body-wall, sex, and anal depressor muscles, whereas the $1.3 \mathrm{~kb}$ internal sequences drove CFP expression only in the pharyngeal muscles and intestine. No expression was observed in the intestinal muscles or the gonadal sheath with either construct, suggesting expression in these tissues are driven by regulatory sequences not included in our rescue constructs.

As expected, the body-wall/anal/sex muscle-specific construct partially restored the Prc phenotype in unc-103(sy557) males (rgEx26) (Table 4). lev-11 expressed from these muscles also rescued the turning behavior defect seen during mating and restored sensitivity to levamisole. Surprisingly, we also found that the pharyngeal muscle/intestine construct partially restored unc103(sy557)-induced protraction (rgEx27) (Table 4). However, unlike the body-wall/anal/sex muscle-specific construct, pharyngeal muscle/intestine expression did not rescue turning behavior or restore levamisole sensitivity but instead partially rescued the vulva location defect (Table 4). Although we did not expect the pharyngeal muscle/intestine-specific expression of lev-11 to af- fect drug resistance or turning behavior, rescue of vulva location and spicule protraction suggested that one or both of these tissues have control over those two aspects of male mating.

In contrast to lev-11(rg1); unc-103(sy557) males, we found that pharyngeal muscle/intestine-specific lev-11(+) can almost completely restore the Prc phenotype for lev-11(rg1); unc103(n1213) males (Table 4). However, the same body-wall/anal/ sex muscle lev-11(+) array that partially rescues lev-11(rg1); unc103(sy557) had no effect on lev-11(rg1); unc-103(n1213) males (Table 4). We conclude that lev-11 (rg1) must be suppressing unc103(sy557) by affecting body-wall, sex, pharyngeal muscles, and/or the intestine, whereas lev-11(rg1) must be suppressing unc-103(n1213) mainly in the pharyngeal muscles and/or intestine.

\section{RNAi knockdown of pharyngeal-specific TNT can suppress unc-103(sy557)-mediated protraction}

Because rescue of unc-103(sy557) and unc-103(n1213)-induced protraction from the pharyngeal muscle/intestine-specific construct was unexpected, we tried to phenocopy the lev-11(rg1)unc-103(lf) genetic interaction by perturbing other molecules involved in muscle contraction. The $r g 1$ mutation in $l e v-11$ is located near the proposed region of TNT binding (Kagawa et al., 1995). TNT is part of the three-subunit complex that tethers troponin $\mathrm{C}$ and troponin I to tropomyosin and is an important regulator of muscle contraction (Filatov et al., 1999). We asked whether there was a specific TNT that responded to the unc103(sy557) lesion. Each of the four TNT proteins was knocked down via RNAi. To verify that any effects observed were specific to unc-103 mutants and not general to all Prc mutants, we assayed two other mutants: one that is suppressed by starvation [lev11(sy558)] and one that is not suppressed by starvation (sy574). We found that reducing tnt-4 in L4 males fully suppressed the unc-103(sy557)-mediated Prc phenotype but not lev-11(sy558) or sy574, demonstrating specificity of reducing TNT-4 to unc-103mediated spicule protraction (Fig. $4 A$ ). tnt- 1 and $t n t-3$ RNAi can significantly reduce the Prc phenotype in lev-11(sy558) and sy574 males, suggesting that although RNAi reduction of these TNT molecules has no strong effect on unc-103(sy557), reducing their expression can suppress protractor contraction caused by defects in other genes.

To determine which cells were responsible for the $t n t-4$ RNAimediated suppression of unc-103(sy557), we constructed the plasmid pTG7, which contains a GFP translational fusion to sequences that span from the gene preceding tnt-4 to the last exon of the smaller tnt-4 isoforms (http://www.wormbase.org). The 
tnt-4::GFP construct expressed only in the pharyngeal muscles of both males and hermaphrodites (Fig. 4B). These data suggest that the pharynx, and probably not the intestine, regulates contraction of the male's sex muscles.

One caveat to the TNT RNAi experiment is that for $u n c-103(s y 557)$, but not for lev-11(sy558) or sy574, males, tnt-4 RNAi may be artificially reducing $t n t-1$, tnt-2, or tnt-3 transcripts in muscle types other than the pharynx. This could occur either by promiscuous interaction of $t n t-4$ RNAi with other $t n t$ transcripts or by indirectly reducing pharyngeal activity, causing food deprivation and a resulting general decrease in other $t n t$ mRNA levels. To test this, we exposed unc-103(sy557) males ( $n=27)$ to a solution containing doublestranded tnt-1, tnt-2, and tnt-3 RNA. We found that $37 \%$ of the males were Prc, which is not comparably different from when unc-103(sy557) males were exposed to solutions containing $t n t-1$, tnt-2, and tnt-3 RNAi separately. From this experiment, we reason that the effect of $t n t-4$ RNAi is specific, and because tnt- 4 is only expressed in the pharynx, we suggest that reducing $t n t$-4- mediated pharyngeal contractions can suppress unc-103(sy557).

\section{The pharyngeal NSM neurons are necessary for lev-11(rg1) suppression of unc-103(lf)}

Because altering pharyngeal muscle proteins can suppress the Prc phenotype in unc-103(sy557) males, we asked which neuron could be a possible mediator of communication between the pharynx and male genitalia. We reasoned that a possible candidate was the pair of NSM neurons located in the pharynx. The NSM neurons have proprioceptive-like endings with synapsefree processes located within the lumen of the pharynx, where bacteria accumulate (Albertson and Thomson, 1976). They also contain processes that run very close to the pseudocoelom. Because of these characteristics, the NSM neurons have been proposed to signal the presence of food to the rest of the animal. We asked whether these neurons were necessary for lev-11(rg1) suppression of the unc-103(sy557) Prc phenotype.

In our experiments, we used a $t p h-1::$ GFP reporter construct (pTG11) to identify the NSM neurons. $t p h-1$ encodes the biosynthetic enzyme, tryptophan hydroxylase, and this gene is expressed in all serotonergic neurons in C. elegans (Sze et al., 2000). lev-11(rg1);unc-103(sy557) and lev-11(rg1); unc-103(n1213) males were isolated at the L3 stage, and the NSM neurons were ablated using a laser microbeam. Ablation of the NSM neurons significantly restored the Prc phenotype in both genetic backgrounds (Fig. 5A), suggesting that the pharynx signals to the genitalia through these neurons.

Previously, others have reported that the NSM neurons are not required for normal pharyngeal pumping behavior in wildtype hermaphrodites (Avery and Horvitz, 1989; Avery et al., 1993). In those studies, NSM-ablated wild-type animals displayed a normal pumping rate and developed normally. Because the NSM neurons are required for $r g 1$-mediated suppression of the Prc phenotype, we asked whether these neurons can regulate pharyngeal pumping rate in lev-11(rg1) and unc-103(sy557) mutant backgrounds (Fig. 5B). The NSM neurons were ablated at the L3 larval stage in both males and hermaphrodites, and animals were assayed for pharyngeal contractions per minute at the L4 stage. In our assays, we found no significant difference in the pumping rate between males and hermaphrodites (data not shown), and as demonstrated previously by Avery and colleagues (Avery and Horvitz, 1989; Avery et al., 1993), wild-type animals with the NSM neurons ablated did not pump at a significantly different rate than the intact controls. However, ablating the NSM neurons in lev-11(rg1) and unc-103(sy557) significantly reduced the pharyngeal contraction rate in both sexes (Fig. 5B). This suggests that in intact animals, the NSM neurons function partially to counteract the decrease in pumping rate caused by mutations in lev-11 and unc-103.

Because of this finding, we considered the hypothesis that although the NSM neurons do not regulate pumping rate during normal foraging behavior, they may control pharyngeal pumping during some other behavioral context. Wild-type males dramatically decrease pumping rate in response to vulva contact (Liu and Sternberg, 1995); because unc-103(lf) and lev-11(rg1) males appear to be in an active and repressed mating state, respectively, we reasoned that mating could be the specific context. To investigate the function of the NSM neurons in more detail, we paired NSM-ablated and intact wild-type males with paralyzed hermaphrodites and observed effects on response to contact, turning, vulva location, spicule insertion, and pharyngeal pumping rate during mating. NSM-ablated animals showed no significant difference in performing the mating substeps compared with 
A

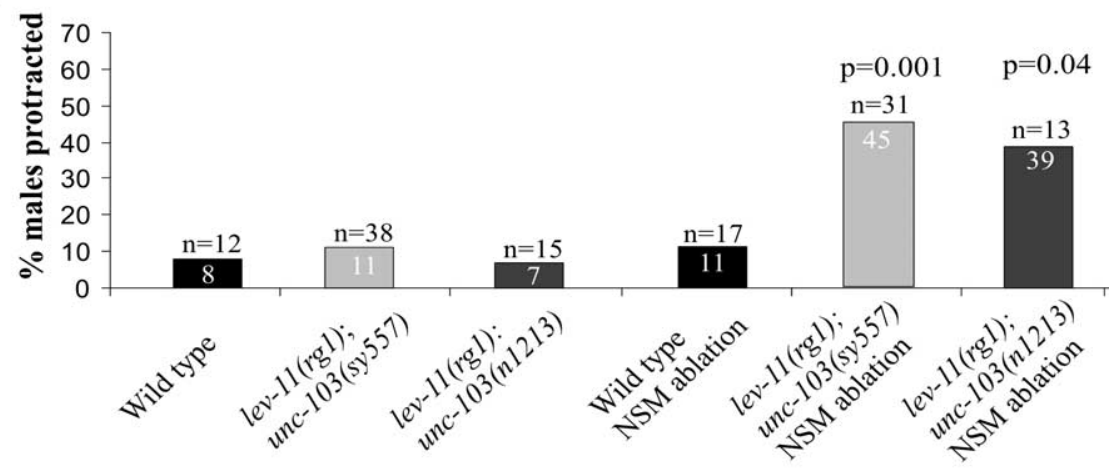

B

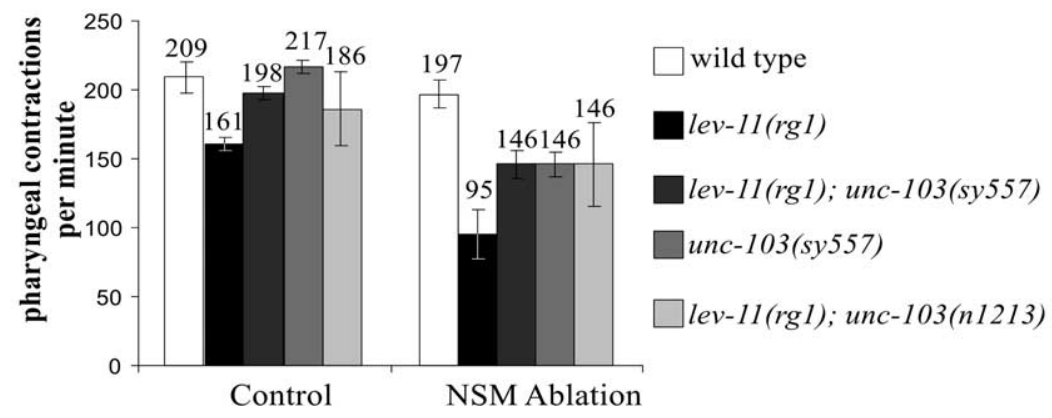

Figure 5. NSM mediates communication between the pharynx and male genitalia. $\boldsymbol{A}$, The effects of ablating the bilateral pair of NSM neurons on the Prc phenotype. The $p$ values are based on the $\chi^{2}$ test. The numbers above the error bars correspond to the actual average numbers of pharyngeal contractions per minute. $\boldsymbol{B}$, The effects on the pharyngeal pumping rate when the NSM neurons are ablated. Error bars represent the SEM.

their intact counterparts (data not shown). However, we did observe a difference in pharyngeal pumping rate during mating. We found that $100 \%(n=11)$ of wild-type males will reduce and eventually stop pharyngeal pumping after vulva contact; in contrast, $55 \%\left(n=11 ; \chi^{2}\right.$ test; $\left.p \leq 0.05\right)$ of NSM-ablated males do not reduce pumping rate in response to vulva contact. These observations suggest that the NSM neurons also function to inhibit pharyngeal pumping rate during mating by controlling pharyngeal contractions in response to vulva contact.

Because the NSM neurons seem to upregulate feeding behaviors to compensate for mutations associated with $l e v-11$ and unc103 , we asked whether loss of UNC-103 function effectively compromises the ability of NSM neurons to control feeding behavior during mating. We found that unc-103(n1213) males show the same defect observed with NSM-ablated wild-type males; $35 \%$ $\left(n=20 ; \chi^{2}\right.$ test; $\left.p \leq 0.05\right)$ of $u n c-103(n 1213)$ males fail to reduce their pumping rate after vulva contact. We then asked whether the addition of the lev-11(rg1) allele could restore this pharyngeal pumping inhibition during mating. In contrast to unc103(n1213), $100 \%\left(n=10 ; \chi^{2}\right.$ test; $\left.p \leq 0.05\right)$ of lev-11(rg1); unc-103(n1213) males repressed pumping after vulva contact. These results are consistent with lev-11(rg1) acting at least partially in the pharynx to suppress unc-103(lf) spicule protraction mutants.

\section{Discussion}

LEV-11 and UNC-103 integrate specific behavioral responses unc-103 encodes a $\mathrm{K}^{+}$channel related to Drosophila seizurelerg and the human herg-encoded delayed inward rectifying voltagegated $\mathrm{K}^{+}$channel (Sanguinetti et al., 1995; Trudeau et al., 1995; Reiner et al., 1999). In humans, perturbations in HERG cause cardiac arrhythmias (Curran et al., 1995), and in flies, ERG mutations cause seizures (Titus et al., 1997; Wang et al., 1997). In contrast, C. elegans mutants lacking UNC103 display precocious sex-muscle contractions as the primary phenotype, despite the broad expression of the channel (Garcia and Sternberg, 2003). Previously, we and others (Liu and Sternberg, 1995; Garcia et al., 2001; Garcia and Sternberg, 2003) have focused on male-specific cells located within the male genitalia to analyze how mating is regulated. Here, we report that male sex muscles are not solely regulated by the circuitry within the tail but are also influenced by muscles and neurons common to both sexes. We found that the spicule insertion step is partially regulated by pharyngeal neurons and muscles (Fig. 6).

The Prc phenotype caused by unc103(n1213) and unc-103(sy557) is suppressed by the rgl allele in the lev-11 tropomyosin gene. This mutation affects all tissue isoforms of tropomyosin (Kagawa et al., 1995). Multiple behaviors such as pharyngeal pumping, egg laying, locomotion, response to contact, male turning, and vulva location are affected by lev-11(rg1); interestingly, spicule muscle contractions during mating is normal. This suggests that muscles other than the spicule protractors can regulate mating. In contrast, the lev-11(sy558) mutation phenocopies unc-103(sy557). This allele demonstrates that tropomyosin can be altered to obtain a specific phenotype without disrupting other behaviors. The sy558 lesion affects an exon found only in bodywall- and sex-muscle isoforms of tropomyosin, therefore its site of action is likely associated with the spicule muscles. We propose that tropomyosin is regulating sex-muscle behaviors by at least two mechanisms: by directly regulating sex-muscle contraction and by indirectly affecting another behavior integrated with male mating.

The effect of lev-11(rg1) on the unc-103(lf) phenotype can be partially alleviated by wild-type tropomyosin in the pharyngeal muscle and intestine. Although wild-type tropomyosin could be acting in either tissue, we believe that the pharynx is the site of action. This is supported by our findings that pharyngeal-specific troponin T (TNT-4) is required for the unc-103-mediated Prc phenotype and that the pharyngeal NSM neurons are partially required for lev-11(rg1) suppression. We suggest that the pharyngeal effect on spicule protraction is attributable to a downregulation of all mating behaviors, rather than a specialized effect on spicule muscles. This is supported by the observations that lev11(rg1) males with wild-type pharyngeal tropomyosin can locate the vulva.

The dominant loss-of-function allele unc-103(sy557) causes a higher penetrance of the Prc phenotype than the unc-103(n1213) deletion allele. In contrast to unc-103(n1213), unc-103(sy557) is not 100\% suppressed by lev-11(rg1); also, the partial suppression is not solely mediated by the NSM neurons. Because unc-103 is expressed in the NSM neurons and the spicule muscles, we hypothesize that the difference between the two unc-103 alleles is attributable to the sy557 protein partially interfering with compensating mechanisms in one or both of these cells. This is supported by our observation that in a lev-11 mutant, introducing 


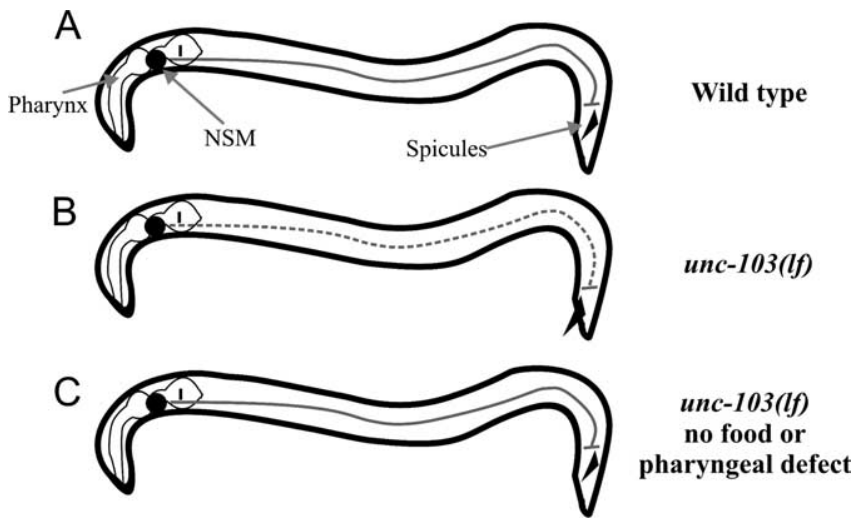

Figure 6. Pharyngeal defects or the absence of food induces the NSM to downregulate excitable cells in the male genitalia. $\boldsymbol{A}$, In wild type, the NSM will downregulate the male genitalia during periods when food is not available. $\boldsymbol{B}$, Loss of unc-103 function in the male genitalia and in the NSM causes the male spicule neurons and muscles to be hyper-excitable. $\boldsymbol{C}$, In unc-103(If) animals, starvation or disrupting the pharyngeal system via mutations can upregulate the NSM neurons to attenuate the male genitalia.

wild-type lev-11 into either the body-wall and sex muscles or into the pharyngeal muscles and the intestine can partially restore the unc-103(sy557)-mediated Prc phenotype, whereas for unc103(n1213), introduction of wild-type tropomyosin into the pharyngeal muscle and intestine, but not in the body-wall and sex muscles, can restore the Prc phenotype. Therefore, lev-11(rg1) may counteract the effects of unc-103(sy557) by affecting the pharyngeal and sex muscles, whereas for unc-103(n1213) males, only the pharynx needs to be altered by lev-11(rg1).

\section{The pharyngeal NSM neurons regulate male sex-muscle behaviors}

Lipton et al. (2004) have described a link between feeding and mate-searching behavior. They found that a $C$. elegans male would stay in environments that include hermaphrodites and food; if there are no hermaphrodites, a well nourished male will leave the food source in search of a mate. However, starved males showed a lower rate of mate searching, suggesting that reduced nutritional status will decrease sex drive. Consistent with the observations of Lipton et al. (2004), we found that unc-103(lf)induced spicule protraction is also food dependent, providing evidence that mating and feeding are regulated by common neurons.

Others have hypothesized that the NSM neurons might sense bacteria in the pharyngeal lumen and signal the presence of food to the rest of the organism by secreting neurotransmitters into the worm's pseudocoelomic space (Albertson and Thomson, 1976). Sawin et al. (2000) showed that they might be a regulator of other behaviors when they observed that food-deprived animals lacking NSM neurons displayed abnormal locomotion when they encountered food. Here, we provide additional evidence for this hypothesis by showing that the NSM neurons affect the penetrance of unc-103(lf) mutations. We propose that the NSM neurons signal pharyngeal physiological status to other areas; specifically, they can suppress mating-specific behaviors when the feeding apparatus is not functioning normally.

Previous work from others determined that the NSM neurons are not essential for normal pumping rate in the presence of bacteria (Avery and Horvitz, 1989). One possible explanation for this observation is that these neurons may regulate pumping in a different environmental context. Our analysis of male mating mutants supports this hypothesis; we found the NSM neurons function to control the wild-type pumping rate during male mating. This is supported by our observation that wild-type males will eventually stop pharyngeal pumping after vulva contact, whereas NSM-ablated males do not. Additionally, the decrease in pumping rate observed in $u n c-103$ (lf) and lev-11(rgl) animals when the NSM neurons are ablated implies they are functioning regularly in mutants. These observations suggest that the NSM neurons can upregulate or downregulate pharyngeal pumping depending on the context.

We speculate that in lev-11(rg1) males, the tropomyosin mutation causes the pharynx to activate the NSM neurons. Consequently, these neurons not only upregulate pharyngeal muscle contractions but also attenuate sex-muscle excitability. This is consistent with our observation that ablating the NSM neurons can partially restore the Prc phenotype in lev-11(rg1); unc103(sy557) and completely restore the Prc phenotype in lev11(rg1); unc-103(n1213) males. Additionally, ablating the NSM neurons in wild type has no effect on spicule insertion, suggesting that the NSM neurons are not required for the proper development of spicule muscles and neurons. Conversely, we note that altering tropomyosin by mutation is distinct from food deprivation in liquid media, because tropomyosin mutants still ingest bacteria during development. This is supported by the observation that reduction in the Prc phenotype associated with food deprivation does not require the NSM neurons (our unpublished observation). Thus, the suppression through food deprivation may be a result of activity downstream of the NSM neurons or in a different pathway. Food deprivation might circumvent NSM requirement by activating the same molecules the NSM neurons activate in response to tropomyosin-mediated pharyngeal muscle contraction. Additionally, we propose that the lev-11(rg1) effect on the pharyngeal musculature does not reduce the Prc phenotype by simply altering pharyngeal pumping rate. Our finding that altering tropomyosin in $u n c-103$ mutants does not reduce pumping rate supports this idea. Furthermore, other mutations that decrease pumping rate, such as eat-18(ad1110) (Raizen et al., 1995; McKay et al., 2004), have no effect on the unc-103(lf)mediated Prc phenotype (data not shown).

The NSM neurons have been reported to contain serotonin, glutamate, the FMRFamide-related protein FLP-4, and the neuropeptide-like proteins NLP-13, NLP-18, and NLP-19 (Albertson and Thomson, 1976; Horvitz et al., 1982; Lee et al., 1999; Sze et al., 2000; Nathoo et al., 2001; Kim and Li, 2004). The interaction between $l e v-11$ ( $r g 1)$ and unc-103(lf) does not require tph-1-encoded tryptophan hydroxylase (our unpublished observation), suggesting that the NSM neurons do not negatively regulate spicule protraction via serotonin. We found this is consistent with work of others that suggest serotonin positively regulates male behaviors such as sex drive, response to hermaphrodites, and ventral turning (Loer and Kenyon, 1993; Lipton et al., 2004). At this time, how the NSM neurons communicate with the male sex muscles is unknown, although it may be through the other neurotransmitters.

Based on our observations, we suggest that the NSM neurons monitor LEV-11 (tropomyosin)-mediated pharyngeal contractions as a proxy for nutrient availability. If the NSM neurons sense normal pharyngeal function, their activity is attenuated; however, if they sense abnormal pumping, they upregulate pharyngeal pumping rate and downregulate excitable cells in the male genitalia (Fig. 6). 


\section{References}

Albertson DG, Thomson JN (1976) The pharynx of Caenorhabditis elegans. Philos Trans R Soc Lond B Biol Sci 275:299-325.

Anyanful A, Sakube Y, Takuwa K, Kagawa H (2001) The third and fourth tropomyosin isoforms of Caenorhabditis elegans are expressed in the pharynx and intestines and are essential for development and morphology. J Mol Biol 313:525-537.

Avery L, Horvitz HR (1989) Pharyngeal pumping continues after laser killing of the pharyngeal nervous system of C. elegans. Neuron 3:473-485.

Avery L, Horvitz HR (1990) Effects of starvation and neuroactive drugs on feeding in Caenorhabditis elegans. J Exp Zool 253:263-270.

Avery L, Bargmann CI, Horvitz HR (1993) The Caenorhabditis elegans unc-31 gene affects multiple nervous system-controlled functions. Genetics 134:455-464.

Bargmann CI, Avery L (1995) Laser killing of cells in Caernorhabditis elegans. In: Methods of cell biology, Vol 48, Caernorhabditis elegans: modern biological analysis of an organism (Shakes DC, ed), pp 225-250. New York: Academic.

Barker DM (1994) Copulatory plugs and paternity assurance in the nematode Caenorhabditis elegans. Anim Behav 48:147-156.

Brenner S (1974) The genetics of Caenorhabditis elegans. Genetics 77:71-94.

Curran ME, Splawski I, Timothy KW, Vincent GM, Green ED, Keating MT (1995) A molecular basis for cardiac arrhythmia: HERG mutations cause long QT syndrome. Cell 80:795-803.

Filatov VL, Katrukha AG, Bulargina TV, Gusev NB (1999) Troponin: structure, properties, and mechanism of functioning. Biochemistry (Mosc) 64:969-985.

Finney DJ (1971) Probit analysis, Ed 3. London: Cambridge UP.

Garcia LR, Sternberg PW (2003) Caenorhabditis elegans UNC-103 ERG-like potassium channel regulates contractile behaviors of sex muscles in males before and during mating. J Neurosci 23:2696-2705.

Garcia LR, Mehta P, Sternberg PW (2001) Regulation of distinct muscle behaviors controls the C. elegans male's copulatory spicules during mating. Cell 107:777-788.

Granato M, Schnabel H, Schnabel R (1994) pha-1, a selectable marker for gene transfer in C. elegans. Nucleic Acid Res 22:1762-1763.

Hodgkin J (1983) Male phenotypes and mating efficiency in Caenorhabditis elegans. Genetics 103:43-64.

Hodgkin JA, Horvitz HR, Brenner S (1979) Nondisjunction mutants of the nematode Caenorhabditis elegans. Genetics 91:67-94.

Horvitz HR, Chalfie M, Trent C, Sulston JE, Evans PD (1982) Serotonin and octopamine in the nematode Caenorhabditis elegans. Science 216:1012-1014.

Kagawa H, Sugimoto K, Matsumoto H, Inoue T, Imadzu H, Takuwa K, Sakube Y (1995) Genome structure, mapping and expression of the tropomyosin gene tmy-1 of Caenorhabditis elegans. J Mol Biol 251:603-613.

Kim K, Li C (2004) Expression and regulation of an FMRFamide-related neuropeptide gene family in Caenorhabditis elegans. J Comp Neurol 475:540-550.

Lee RY, Sawin ER, Chalfie M, Horvitz HR, Avery L (1999) EAT-4, a homolog of a mammalian sodium-dependent inorganic phosphate cotransporter, is necessary for glutamatergic neurotransmission in Caenorhabditis elegans. J Neurosci 19:159-167.

Lee RYN, Lobel L, Hengartner M, Horvitz HR, Avery L (1997) Mutations in the $\alpha 1$ subunit of an L-type voltage-activated $\mathrm{Ca}^{2+}$ channel cause myotonia in Caenorhabditis elegans. EMBO J 16:6066-6076.

Lewis JA, Wu CH, Berg H, Levine JH (1980) The genetics of levamisole resistance in the nematode Caenorhabditis elegans. Genetics 95:905-928.

Lipton J, Kleemann G, Ghosh R, Lints R, Emmons SW (2004) Mate searching in Caenorhabditis elegans: a genetic model for sex drive in a simple invertebrate. J Neurosci 24:7427-7434.

Liu K, Sternberg PW (1995) Sensory regulation of male mating behavior in Caenorhabditis elegans. Neuron 14:79-89.

Loer CM, Kenyon CJ (1993) Serotonin-deficient mutants and male mating behavior in the nematode Caenorhabditis elegans. J Neurosci 12:5407-5417.

McKay JP, Raizen DM, Gottschalk A, Schafer WR, Avery L (2004) eat-2 and eat-18 are required for nicotinic neurotransmission in the Caenorhabditis elegans pharynx. Genetics 166:161-169.

Mello CC, Kramer JM, Stinchcomb D, Ambros V (1991) Efficient gene transfer in C. elegans: extrachromosomal maintenance and integration of transforming sequences. EMBO J 10:3959-3970.

Moghal N, Garcia LR, Khan LA, Iwasaki K, Sternberg PW (2003) Modulation of EGF receptor-mediated vulva development by the heterotrimeric G-protein Galphaq and excitable cells in C. elegans. Development 130:4553-4566.

Nathoo AN, Moeller RA, Westlund BA, Hart AC (2001) Identification of neuropeptide-like protein gene families in Caenorhabditis elegans and other species. Proc Natl Acad Sci USA 98:14000-14005.

Ohtsuki I, Maruyama K, Ebashi S (1986) Regulatory and cytoskeletal proteins of vertebrate skeletal muscle. Adv Protein Chem 38:1-67.

Ono S, Baillie DL, Benian GM (1999) UNC-60B, an ADF/cofilin family protein, is required for proper assembly of actin into myofibrils in Caenorhabditis elegans body wall muscle. J Cell Biol 145:491-502.

Park EC, Horvitz HR (1986) Mutations with dominant effects on the behavior and morphology of the nematode Caenorhabditis elegans. Genetics 113:821-852.

Petersen CI, McFarland TR, Stepanovic SZ, Yang P, Reiner DJ, Hayashi K, George AL, Roden DM, Thomas JH, Balser JR (2004) In vivo identification of genes that modify ether-a-go-go-related gene activity in Caenorhabditis elegans may also affect human cardiac arrhythmia. Proc Natl Acad Sci USA 101:11773-11778.

Raizen DM, Lee RY, Avery L (1995) Interacting genes required for pharyngeal excitation by motor neuron MC in Caenorhabditis elegans. Genetics 141:1365-1382.

Reiner DJ, Newton EM, Tian H, Thomas JH (1999) Diverse behavioural defects caused by mutations in Caenorhabditis elegans unc-43 CaM kinase II. Nature 402:199-203.

Sanguinetti MC, Jiang C, Curran ME, Keating MT (1995) A mechanistic link between an inherited and an acquired cardiac arrhythmia: HERG encodes the $\mathrm{I}_{\mathrm{Kr}}$ potassium channel. Cell 81:299-307.

Sawin ER, Ranganathan R, Horvitz HR (2000) C. elegans locomotory rate is modulated by the environment through a dopaminergic pathway and by experience through a serotonergic pathway. Neuron 26:619-631.

Schnabel H, Schnabel R (1990) An organ-specific differentiation gene, pha-1, from Caenorhabditis elegans. Science 250:686-688.

Smillie LB (1979) Structure and functions of tropomyosins from muscle and nonmuscle sources. Trends Biochem Sci 4:151-155.

Sulston JE, Albertson DG, Thomson JN (1980) The Caenorhabditis elegans male: postembryonic development of nongonadal structures. Dev Biol 78:542-576.

Sze JY, Victor M, Loer C, Shi Y, Ruvkun G (2000) Food and metabolic signalling defects in a Caenorhabditis elegans serotonin-synthesis mutant. Nature 403:560-564.

Taylor EW (1979) Mechanism of actomyosin ATPase and the problem of muscle contraction. CRC Crit Rev Biochem 6:103-164.

Thomas JH (1990) Genetic analysis of defecation in Caenorhabditis elegans. Genetics 124:855-872.

Titus SA, Warmke JW, Ganetzky B (1997) The Drosophila erg K ${ }^{+}$channel polypeptide is encoded by the seizure locus. J Neurosci 17:875-881.

Trent C, Tsung N, Horvitz HR (1983) Egg-laying defective mutants of the nematode Caenorhabditis elegans. Genetics 104:619-647.

Trudeau MC, Warmke JW, Ganetzky B, Robertson GA (1995) HERG, a human inward rectifier in the voltage-gated potassium channel family. Science 269:92-95.

Wang XJ, Reynolds ER, Deak P, Hall LM (1997) The seizure locus encodes the Drosophila homolog of the HERG potassium channel. J Neurosci 17:882-890.

Ward S, Carrel JS (1979) Fertilization and sperm competition in the nematode Caenorhabditis elegans. Dev Biol 73:304-321.

White JG, Southgate E, Thomson JN, Brenner S (1986) The structure of the nervous system of Caenorhabditis elegans. Philos Trans R Soc Lond B Biol Sci 14:1-340.

Wicks SR, Yeh RT, Gish WR, Waterston RH, Plasterk RHA (2001) Rapid gene mapping in Caenorhabditis elegans using a high density polymorphism map. Nat Genet 28:160-164. 